\title{
Carta Interpretativa do Solo do Mioceno da Charneca do Ribatejo
}

\author{
Nuno Neves*, Teresa Afonso, Alfredo Gonçalves Ferreira, Ana \\ Cristina Gonçalves, Nuno Ribeiro e Susana Dias
}

Sumário. O solo é um factor fundamental na sustentabilidade de um sistema florestal. As cartas interpretativas de uso do solo indicam a sua adequação a uma dada utilização. Este potencial pode ser avaliado com recurso a características-diagnóstico de cada unidade de solo, que pela sua presença ou ausência influenciam o seu potencial produtivo. O objectivo deste estudo foi o desenvolvimento de um modelo de adequação de uma característica diagnóstico (FERREIRA et al., 2001), de aptidão potencial do solo para o sobreiro, em ambiente de sistemas de informação geográfica, considerando como variáveis independentes, a topografia e a perda e deposição potencial de solo, expressas através dos índices de Erosão Hídrica Estrutural (EHE) e de Deposição Estrutural Potencial (DEP). Esta metodologia permitiu a obtenção de uma carta interpretativa do solo, discriminando a referida classe em cinco. A análise estatística evidencia aderência do modelo à realidade utilizando como indicador o peso anidro de cortiça por unidade de área descortiçada $\left(\mathrm{PCM}^{2}\right)$, dando origem a uma carta com mais detalhe.

Palavras-chave: SIG; características-diagnóstico, EHE; DEP; $\mathrm{PCM}^{2}$

Soil Interpretive Map of the Miocene of the Charneca do Ribatejo

Abstract. Soil is a fundamental factor in the sustainability of a forest system. The interpretive soil use charts point out the suitability for a given use. The soil potential can be appraised referring to diagnostic-characteristics of each soil unit, which due to its presence or absence may influence the soil use suitability. This study's purpose is the development of a model of adequacy of one of the diagnostic-characteristic (FERREIRA et al., 2001), for cork oak. This development takes place in a geographic information system environment, considering topography, potential soil loss and deposition a sin dependent variables. These variables are expressed through indexes of Structural Water Erosion

*Departamento de Paisagem, Ambiente e Ordenamento, Universidade de Évora, Colégio Luís António Verney, 7000-671 ÉVORA, Portugal

E-mail: alfredo@uevora.pt

This is an Open Access article distributed under the terms of the Creative Commons Attribution License (https://creativecommons.org/licenses/by/4.0), which permits unrestricted use, distribution, and reproduction in any medium, provided the original work is properly cited. 
(EHE) and Potential Structural Deposition (DEP). This method ology opened the way to the creation of a soil capability map, where the above referred diagnostic-characteristicis split in five classes. The statistical analysis shows the model's adherence to reality, using then ahydrous weight of cork $\left(\mathrm{PCM}^{2}\right)$ peruni to feedbarked area as an indicator. The present methodology produces a more detailed soil capability class map.

Key words: GIS; diagnostic-characteristics; EHE; DEP; $\mathrm{PCM}^{2}$

\section{Carte Interprétative du Sol du Miocène de la Charneca do Ribatejo}

Résumé. Le sol est un facteur clé de la durabilité d'un système forestier. Les tableaux' interprétatifs de l'utilisation des terres indiquent leur pertinence pour une certaine utilisation. Ce potentiel peut être évalué à l'aide des caractéristiques-diagnostiques de chaque unité de sol qui, par sa présence ou absence, influencent leur potentiel de production. Le but de cette étude c'est de développer un modèle d'adéquation d'une caractéristique-diagnostique (FERREIRA et al., 2001), de l'adéquation potentielle du sol au chêne-liège. Ce modèle doit prendre place dans un environnement de systèmes d'information géographique. Pour cette étude ont a considéré comme variables indépendantes la topographie, la perte et le dépôt potentiels du sol, exprimés à travers les indices d'érosion structurelle par l'eau (EHE) et de dépôt potentiel structurel (DEP). Cette méthodologie a permis d'obtenir une carte interprétative du sol, discriminant la classe référencée sur cinq. L'analyse statistique prouve l'adhésion du modèle à la réalité, en utilisant comme indicateur le poids anhydre du liège par unité de surface exploitée $\left(\mathrm{PCM}^{2}\right)$, donnant ainsi lieu à une carte plus détaillé que les antérieures.

Mots-clés: SIG; caractéristiques- diagnostique; EHE; DEP; $\mathrm{PCM}^{2}$ 


\section{Introdução}

A sustentabilidade de um sistema florestal é condicionada por vários factores, dos quais o potencial do solo para suportar vida tem um papel primordial (ÖZHAN et al., 2005; SCHOENHOLTZ et al., 2000; SILVA, 1999; RICHARDSON et al., 1999; JENNY, 1983; WISCHMEIER e SMITH, 1965; KOHNKE e BERTRAND, 1959).

A formação do solo resulta da combinação de factores de estado, variáveis independentes, cuja primeira identificação (DOKUCHAEV, 1899) e quantificação (JENNY, 1941) é desenvolvida na equação de formação de solo (Eq. 1), onde; S solo, cl, clima, p, material orgânico, o, organismos, r, relevo e t, tempo (JENNY, 1941).

$$
S=f(c l, p, o, r, t)
$$

As cartas interpretativas de uso do solo identificam a adequação da cada unidade do solo, taxon, a uma dada utilização. As características transversais a diferentes taxa, características-diagnóstico, determinam o seu comportamento e tipo de utilização, originando cartas de aptidão, para diversas utilizações, nomeadamente para o sobreiro (FERREIRA et al., 2001).

O fenómeno da erosão-deposição, quer hídrica ou eólica, influencia potencial produtivo do solo, são processos cumulativos, por isso a vulnerabilidade do solo aumenta com o seu progresso (SCHOENHOLTZ et al., 2000; SILVA, 1999). A produtividade das espécies florestais depende do clima e da capacidade do solo para armazenar água e nutrientes minerais, trocas gasosas, actividade biológica e potencial para o desenvolvimento das raízes (SCHOENHOLTZ et al., 2000; RICHARDSON et al., 1999). A capacidade produtiva de um dado local é avaliada pela classe de qualidade da estação, que pela sua formulação se assume independente da gestão (SCHOENHOLTZ et al., 2000; RICHARDSON et al., 1999). A classe de qualidade da estação para o sobreiro, Quercus suber L., é definida pelo peso anidro por unidade de superfície descortiçada, $\mathrm{PCM}^{2}$ (obtido por pesagem após 72 horas em estufa a $103^{\mathrm{a}} \mathrm{C} \pm 3^{\circ} \mathrm{C}$ ) de sobreiros com cortiça amadia (MONTERO, 1988).

A análise da carta de características-diagnóstico para o sobreiro, no Mioceno da Charneca do Ribatejo, revela a necessidade de maior detalhe para a classe, sem características-diagnóstico. O objectivo deste estudo é o desenvolvimento de uma metodologia de interpretação da carta de solos, em função de variáveis topográficas, que permitam maior detalhe. A abordagem deste estudo centrou-se no desenvolvimento de uma nova aproximação metodológica, em sistemas 
de informação geográfica, considerando como variáveis independentes a perda e deposição potencial de solo. A aderência do modelo foi efectuada com uma base no peso anidro de cortiça, por unidade de superfície descortiçada, dados de parcelas permanentes, como indicador da classe de qualidade da estação.

\section{Modelo de aptidão potencial para o sobreiro no Mioceno}

\section{Caracterização da área de estudo}

O trabalho foi desenvolvido na formação do Mioceno do Ribatejo, na área de actuação da Associação de Produtores Florestais do Concelho de Coruche e Limítrofes, compreendendo às Zonas de Intervenção Florestal da Calha do Grou, Ribeira da Erra, Ribeiras da Lamarosa e Magos, Ribeira do Divor e Baixo Sorraia. (Figura 1). A área de estudo enquadra-se na Charneca do Mioceno, zona ecológica submediterrânea (ALBUQUERQUE, 1954), com topografia do tipo mesa com vales abertos, onde a altitude que não ultrapassa 150m (GOMES, 1969). Nos terraços do quaternário a evolução do solo é marcada, pelo factor de estado tempo ( $\mathrm{t}$ ) e na charneca do Miocénico pelos factores de estado, material originário $(\mathrm{p})$ e relevo $(\mathrm{r})$.

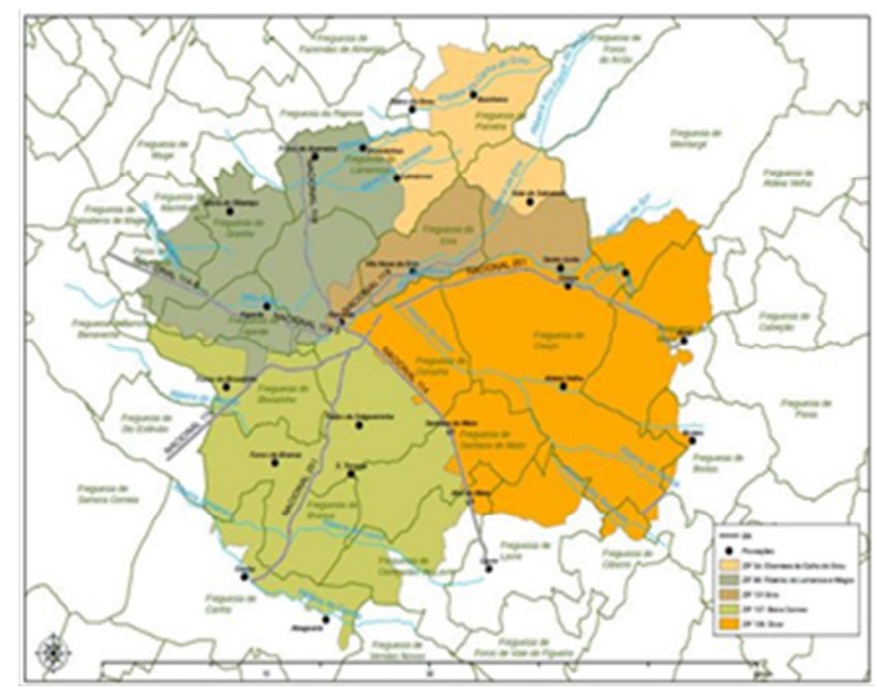

Figura 1 - Localização da área de estudo 
O clima é caracterizado por uma precipitação média anual de 650 a $700 \mathrm{~mm}$, com 60 a 70 dias de chuva por ano, ocorrendo 60 a 70\% entre Novembro e Março, e uma temperatura média anual de 15 a $18^{\circ} \mathrm{C}$. O mês mais quente é Julho, com uma temperatura média de $22.5^{\circ} \mathrm{C}$ e o mais frio é Janeiro, com uma temperatura média de $9^{\circ} \mathrm{C}$ (CIDFCIBCS, 2015). Os materiais originários do solo resultam, predominantemente, de formações arenosas mais ou menos consolidadas do Mioceno, arenitos com cimentos ferruginosos ou argilosos e gredas (GOMES, 1969). O coberto vegetal, nas encostas e vales, é predominantemente montado de sobreiro (Quercus suber L.) por vezes em consociação com pinheiro bravo (Pinus pinasterAiton) e ou pinheiro manso (Pinus pinea L.). Nas zonas planas de topo, mesas, rareia o montado e ocorre o eucalipto (Eucaliptus globulus L.) e o pinheiro bravo (GOMES, 1969).

A área de estudo abrange cerca de 152000 ha, correspondendo às zonas produtivas do ponto de vista florestal, excluindo afloramento rochosos, área social e zonas sujeitas a inundação. A característica diagnóstico de FERREIRA et al. (2001), sem, características-diagnóstico, correspondendo a cerca de $76 \%$ da área (Quadro 1). A área classificada como Scd (Quadro 1) abrange diferentes posições no relevo, onde os fenómenos de perda, deposição do solo e humidade, que influenciam o potencial produtivo do solo, não são evidenciados. Conforme foi referido, o sobreiro ocorre predominantemente nas encostas e vales, rareando nas encostas mais erosionadas e no topo, devido à degradação do solo (GOMES, 1969).

Quadro 1 - Características diagnóstico

\begin{tabular}{|l|r|r|}
\hline \multirow{2}{*}{ Característica-Diagnóstico } & \multicolumn{2}{c|}{ Área } \\
\cline { 2 - 3 } & \multicolumn{1}{|c|}{ Ha } & \multicolumn{1}{c|}{$\%$} \\
\hline Armazenamento_água (Aa) & $8,540.39$ & 5.6 \\
\hline Calcário_activo (Cal) & $1,454.34$ & 1.0 \\
\hline Descontinuidade_textural (Dt) & 366.63 & 0.2 \\
\hline Drenagem_externa (De) & $6,007.89$ & 4.0 \\
\hline Drenagem_interna (Di) & $18,143.54$ & 11.9 \\
\hline Profundidade_expansível (Pe) & $1,217.08$ & 0.8 \\
\hline Sem característica-diagnóstico (Scd) & $116,265.11$ & 76.5 \\
\hline Total & $151,994.98$ & 100.0 \\
\hline
\end{tabular}


Desenvolvimento do modelo

A metodologia foi desenvolvida para as áreas classificadas como Scd, de modo a possibilitar a discriminação de novas, características-diagnóstico (Figura 2), com melhor aderência à realidade. $\mathrm{O}$ modelo de aptidão para o sobreiro considerou como variáveis independentes a Erosão Hídrica Estrutural (EHE) e a Deposição Estrutural Potencial (DEP). Estas duas variáveis foram derivadas do modelo digital de elevação (MDE). O modelo foi desenvolvido em ambiente de sistemas de informação geográfica, com o programa ArcGIS versão 10.6.8321 (ESRI, 1999/2017).

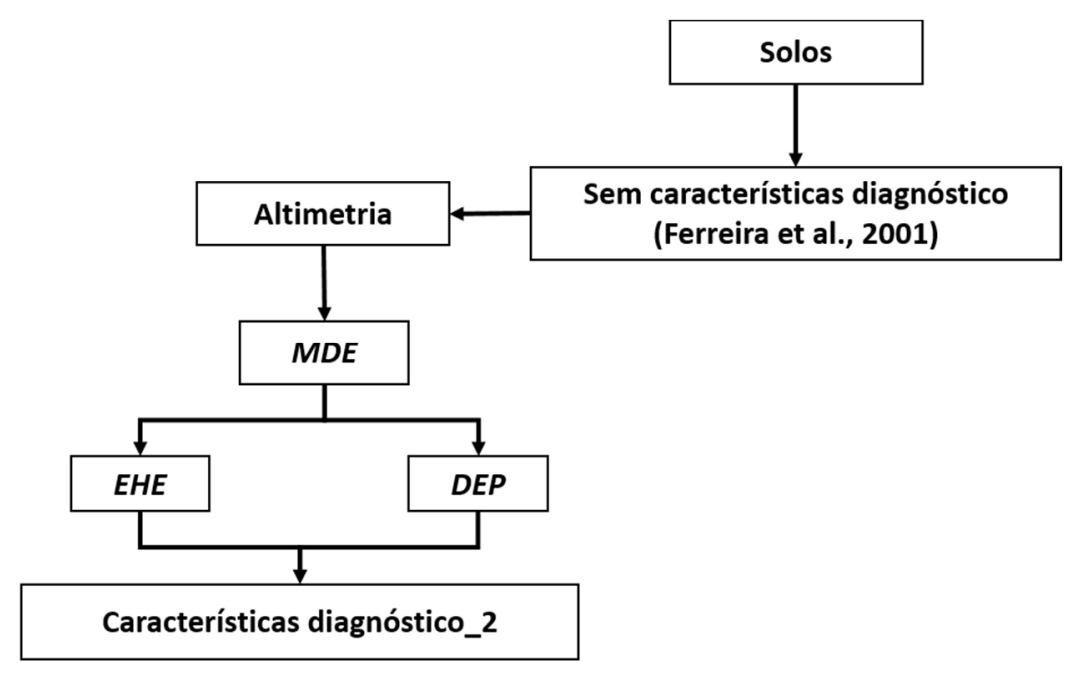

Figura 2 - Representação esquemática do modelo cartográfico

\section{Modelo Digital de Elevação, MDE}

O modelo digital de elevação (MDE) desenvolvido a partir das cartas de altimetria à escala 1:25.000 (Carta Militar de Portugal - Série M888), por uma técnica interpolativa iterativa, "iterativefinite diference interpolationtechnique," (WAHBA, 1990), que permite estimar de valores numa perspectiva contextual decorrente de um processo multiresolução adaptativo. Esta técnica interpolativa iterativa, a partir de bases informativas com frequências de observação altimétrica relativamente baixas, permite estimar de forma mais 
adequada o comportamento "funcional" do relevo em processos de escoamento. Este foi derivado a partir da aproximação interpolativa "drainageenforcement", que identifica e remove as depressões do terreno, susceptíveis de afectar a modelação do processo de escoamento, dando origem ao um modelo digital de elevação mais "hidrologicamente correcto".

O processo de interpolação "topo toraster" com drenagem forçada, considera a integração de diversas fontes de observação altimétrica (e.g. isolinhas, pontos cotados), bem como outro tipo de entidades, destinadas a especificar as condições de interpolação e os resultados finais. Num contexto de aplicação, em que é fundamental a concordância entre diferentes fontes de informação, é utilizada uma base informativa da rede hidrográfica, devidamente orientada, de forma a condicionar a definição do $M D E$ às linhas de água, assumidas como correctamente definidas espacialmente (NEVES et al., 2001).

\section{Erosão Hídrica Estrutural, EHE}

A Erosão Hídrica Estrutural (EHE, Eq. 2, Figura 3) é um índice multiplicativo centrado na avaliação da força gravítica e da friç̧ão do atrito, integrando ainda um indicador potencial de profundidade do solo (Tc) e do indicador de atrito (Rp), calculados por operações de álgebra de mapas.

$$
\mathrm{EHE}=\mathrm{DF} \times \mathrm{Tc} \times \mathrm{Rp}=\tan ^{-1}\left(\frac{\mathrm{hp}}{\left(\sin \left(90^{\circ}-a \mathrm{p}\right)\right) \times 100}\right) \times \mathrm{Tc} \times \mathrm{Rp}
$$

O declive força (DF) é um factor primordial em processos de erosão decorrentes do escoamento superficial. É uma métrica independente do atrito e da erosão, resultante da dissipação da energia provocada pela interação da força gravítica e da força de atrito e do impacto hidráulico, decorrentes da variação do declive, orientação da encosta e erodibilidade do solo, calculada através de uma relação entre a soma ponderada dos valores de altitude drenante para cada ponto (hp), exprimindo a contribuição cumulativa dos valores de altitude relativa de todas as células drenantes para cada local; e a soma ponderada dos ângulos de inclinação (ap), exprimindo em cada local a contribuição cumulativa dos declives de todas as células drenantes para cada local. Destas variáveis deriva a fórmula $D F=\tan ^{-1}\left(\frac{\mathrm{hp}}{\left(\sin \left(90^{\circ}-\mathrm{ap}\right)\right) \times 100}\right)$, que é uma métrica adimensional proporcional ao trabalho da força gravítica.

$\mathrm{O}$ indicador de atrito resultante da reacção ao peso (Rp) é expresso como o cosseno do ângulo de inclinação, a., decorrendo da aplicação directa das leis do 
movimento, considerando a deslocação de materiais líquidos num processo de escoamento superficial.

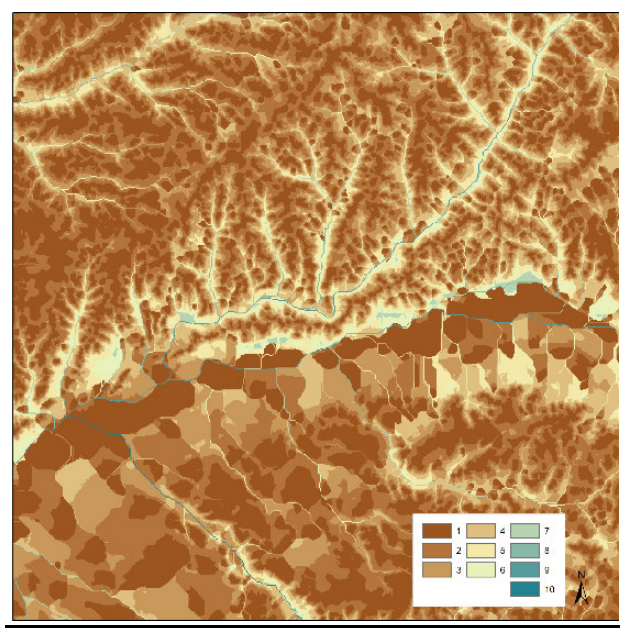

Figura 3 - Cálculo da Erosão Hídrica Estrutural (EHE)

No modelo EHE foi desenvolvida uma aproximação centrada na combinação de duas possibilidades ou modelos alternativos: i) acumulação concentrada máxima, resultante da aplicação de uma função de acumulação de escoamento segundo o modelo clássico D8 dos valores do declive (JENSON e DOMINGUE, 1988); ii) acumulação difusa máxima, resultante da aplicação de uma função de comprimento de encosta (downs tream) sobre um MDE invertido (NEVES et al., 2001). A utilização combinada das duas aproximações permite uma caracterização do processo de escoamento, que se considera reflectir de forma mais adequada o potencial máximo de escoamento.

A métrica EHE é condicionada ao intervalo $\left[0^{\circ}, 90^{\circ}\right]$ pelo inverso da função tangente e permite a obtenção de valores similares aos resultados do cálculo normal do declive em graus (Eq. 2).

\section{Deposição Estrutural Potencial, DEP}

O índice de deposição estrutural potencial (DEP, Eq. 3, Figura 4) é uma métrica de normalização de uma estimativa da deposição de materiais provenientes de escoamento hídrico superficial. A estrutura conceptual da métrica baseia-se no estabelecimento de uma relação normalizada entre o 
transporte potencial de materiais e a diminuição da velocidade de escoamento, resultantes da diminuição do declive ao longo da encosta e do valor do declive local (NEVES et al., 2001). Este índice é calculado em função de três variáveis; a integração da acumulação concentrada e difusa dos valores de EHE (IA_EHE), da integração da acumulação concentrada e difusa dos valores do declive (IA_Slope) e do declive (Slope). Considera-se que a quantidade de material erodido é função dos valores da erosão hídrica, calculado pelo EHE.

$$
\text { DEP }=\tan ^{-1} \frac{\text { IA_EHE }}{\text { IA_Slope }} \cdot 57,296 \cdot \cos ^{2} \text { Slope }(r a d)
$$

A integração dos valores de EHE (IA_EHE) e do declive (IA_Slope) ao longo das encostas é efectuada com um modelo de transporte similar ao EHE, que integra duas aproximações de escoamento, a acumulação concentrada e a difusa. O quociente entre os valores de IA_EHE e IA_Slope, indica que na base das encostas existe a continuação do incremento de IA_EHE e uma diminuição de IA_Slope, contribuindo para um aumento progressivo de DEP. O quociente IA_EHE/IA_Slope é afectado pelo inverso da função tangente e multiplicado por 57,296 (factor de conversão radianos-graus), permitindo a sua expressão normalizada em graus, à semelhança do índice EHE (NEVES et al., 2001).

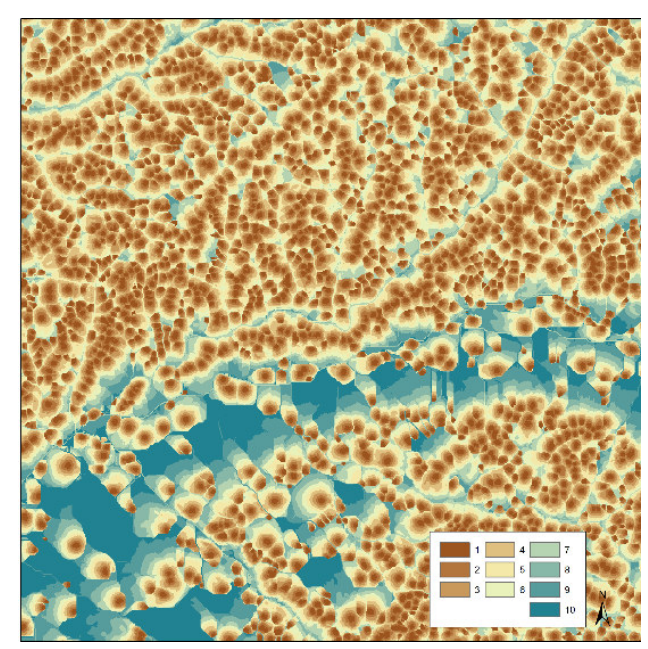

Figura 4 - Cálculo da Deposição Estrutural Potencial (DEP)

Como os processos de deposição não possuem unicamente uma dimensão global (i.e., resultante da acumulação ao longo da bacia), mas também uma 
dimensão local resultante do cálculo focal do declive (Slope, calculo focal 3×3), o quociente de base é multiplicado pelo cosseno do declive local, que pode ser entendido também como um factor de atrito e consequente deposição (NEVES et al., 2001).

\section{Modelo de aptidão para o sobreiro}

As novas, características-diagnóstico foram desenvolvidas a partir de análise de clusters, pericial e reclassificação. A análise de clusters consiste no agrupamento de um conjunto de variáveis, num número restrito de subconjuntos, clusters, em que os elementos de cada um deles são mais semelhantes entre si do que com os outros clusters (MERREL e DIAZ, 2015). O método Isoclusters é uma classificação não supervisionada exploratória, em que o algoritmo isso ("iterative self-organizing") promove o agrupamento de células no espaço multidimensional, constituído por um conjunto de variáveis, layers, em ambiente SIG (BALL e HALL, 1965). Na criação de Isoclusters é utilizada uma abordagem iterativa na optimização do processo de agrupamento, "migrating means technique" (BALL e HALL, 1965). A classificação não supervisionada tem como pressuposto que todas as variáveis apresentarem um domínio similar. Assim, EHE e DEP foram normalizadas pela Eq. 4.

$$
\text { Valor normalizado }=\frac{\text { Valor original-Média zonal }}{\text { Desvio padrão zonal }}
$$

Tratando-se de uma aproximação iterativa, que depende do número de classes iniciais, baseia-se em parâmetros de controlo, nomeadamente número de células mínimo em cada cluster e intervalo da amostra. As células são sucessivamente agrupadas, através da minimização das distâncias euclidianas, ao valor médio em cada classe do espaço multidimensional de variáveis. O número final de classes representa uma medida de estabilidade de pertença de cada célula a cada isocluster, garantindo que os parâmetros são respeitados e que cada célula é classificada (BALL e HALL, 1965).

Neste estudo considerou-se um número inicial de 100 classes, que resultaram em 49 classes optimizadas. A classificação de isoclusters foi sujeita a processos de generalização, visando eliminar áreas sem significado espacial, inferior a 1ha, e reconstituição através da mínima distância a um valor classificado, do qual resultaram 45 isoclusters. Este tema matricial foi posteriormente convertido em formato vectorial. 
A análise pericial foi efectuada com o tema 45 isoclusters, em formato vectorial, ao qual foram adicionados os valores médios de EHE e DEP. Os valores médios de EHE e DEP foram reclassificados em três classes: baixa, média e alta. As classes de erosão potencial, derivadas de EHE, correspondem ao terço inferior, baixa (A); ao terço central, média (B); e ao terço superior, alta (C). De modo semelhante, as classes de deposição potencial, correspondem ao terço inferior dos valores de DEP, baixa (A); ao terço central, média (B); e ao terço superior, alta (C).

A partir de uma operação de álgebra de mapas (Union), dos temas isoclusters e características-diagnóstico, resultaram cinco novas, característicasdiagnóstico (Quadro 2): i) Toalha freática superficial (Tfr), que corresponde a valores baixos (A) de EHE e DEP, indicam relevo aplanado, com fraco potencial de escoamento, que associado à presença de um horizonte câmbico, perfil $\mathrm{ABcC}$, potencia a excesso temporário de água no perfil do solo; ii) Acumulação (Sd), que corresponde a valores médios de EHE (B) e altos de DEP (C), que associado à presença de um horizonte câmbico, perfil $\mathrm{ABcC}$, fases espessa e pedregosa, deposição de materiais grosseiros no horizonte superficial, com o aumento da sua espessura e macro porosidade, boa drenagem interna; iii) Alagamento ocasional (Ao), que corresponde a valores baixos (A) de EHE e DEP, indicam relevo aplanado com fraco potencial de escoamento, ocorrendo em solos derivados de material originário consolidado, perfil $\mathrm{AC} / \mathrm{ABCC}$, potenciam a acumulação temporária de água à superfície do solo; iv) Perda de solo (Er), que corresponde a valores de EHE altos (C) e de DEP médios (B), solos com material originário consolidado, perfil $\mathrm{AC} / \mathrm{ABCC}$, em que a erosão tem domínio sobre a deposição, com consequente diminuição da espessura do perfil do solo; v) Sem características-diagnóstico ( $\mathrm{Scd}$ ), solos sem as características anteriores.

Quadro 2 - Novas características diagnóstico

\begin{tabular}{|l|l|c|c|}
\hline \multicolumn{1}{|c|}{ Perfil } & \multicolumn{1}{|c|}{ Horizonte } & EHE/DEP & $\begin{array}{c}\text { Característica } \\
\text { Diagnóstico }\end{array}$ \\
\hline $\mathrm{AB}_{\mathrm{C}} \mathrm{C}$ & Com Horizonte $\mathrm{B}_{\mathrm{c}}$ & $\mathrm{AA}$ & $\mathrm{Tft}$ \\
\hline $\mathrm{AB}_{\mathrm{c}} \mathrm{C}(\mathrm{e}, \mathrm{p})$ & Com Horizonte $\mathrm{B}_{\mathrm{c}}$, fases espessa e pedregosa & $\mathrm{BC}$ & $\mathrm{Sd}$ \\
\hline $\mathrm{AC} / \mathrm{AB}_{\mathrm{c}} \mathrm{C}$ & Com Material Originário consolidado & $\mathrm{AA}$ & $\mathrm{Ao}$ \\
\hline $\mathrm{AC} / \mathrm{AB}_{\mathrm{c}} \mathrm{C}$ & Com Material Originário consolidado & $\mathrm{CB}$ & $\mathrm{Er}$ \\
\hline $\mathrm{AC} / \mathrm{AB}_{\mathrm{c}} \mathrm{C}$ & Com Material Originário não consolidado & $\mathrm{AA}$ & $\mathrm{Sd}$ \\
\hline Todos os restantes & & $\mathrm{Scd}$ \\
\hline
\end{tabular}


No caso dos complexos de solo a característica é considerada se for $\geq$ a $50 \%$ da área. O novo tema, características-diagnóstico (Figura 5), após procedimentos de generalização cartográfica, permitiu discriminar a área associada à classe sem características-diagnóstico de FERREIRA et al. (2001) (Figura6). As características-diagnóstico definidas neste estudo identificaram cerca de $44 \%$ da área com características específicas para desenvolvimento do sobreiro, das quais as mais representativas em termos de área são a perda de solo (Er) com 19\% e acumulação (Sd) com 12\% (Quadro 3).

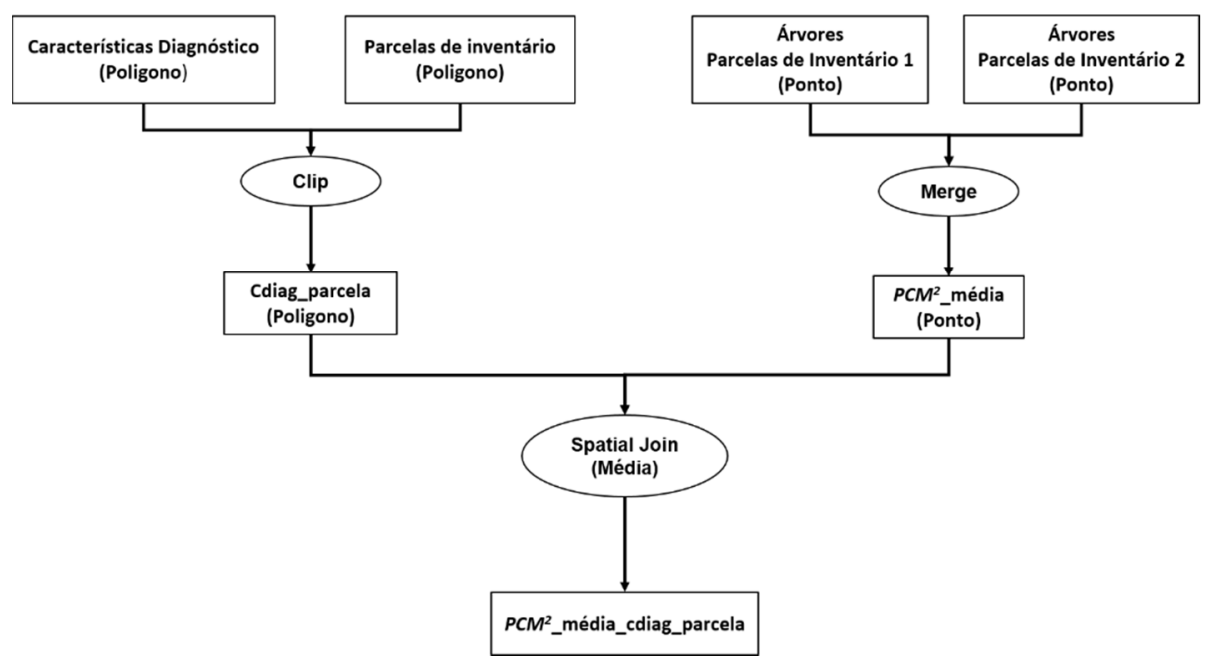

Figura 5 - Fluxograma da carta PCM2_médio_características-diagnósticoparcela

Quadro 3 - Área e percentagem da Reclassificação

\begin{tabular}{|l|r|r|}
\hline \multirow{2}{*}{ Reclassificação } & \multicolumn{2}{|c|}{ Área } \\
\cline { 2 - 3 } & \multicolumn{1}{|c|}{ ha } & \multicolumn{1}{c|}{ \% } \\
\hline Tft & $9,699.87$ & 8.3 \\
\hline Sd & $14,139.17$ & 12.2 \\
\hline Ao & $4,370.81$ & 3.8 \\
\hline Er & $22,089.80$ & 19.0 \\
\hline Scd & $65,965.46$ & 56.7 \\
\hline Total & $116,256.11$ & 100.0 \\
\hline
\end{tabular}


a)

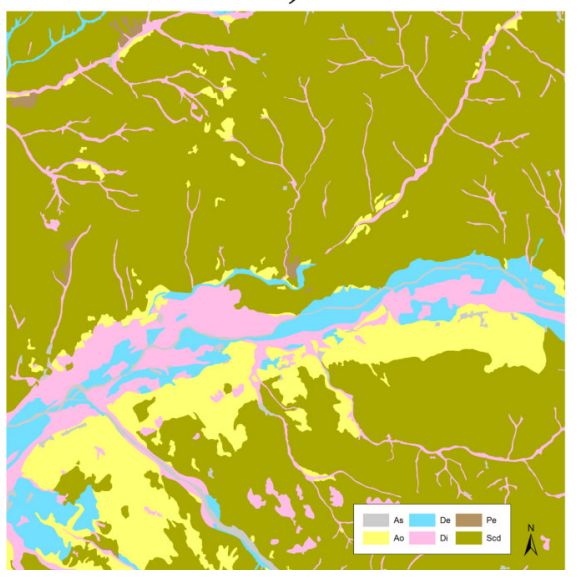

b)

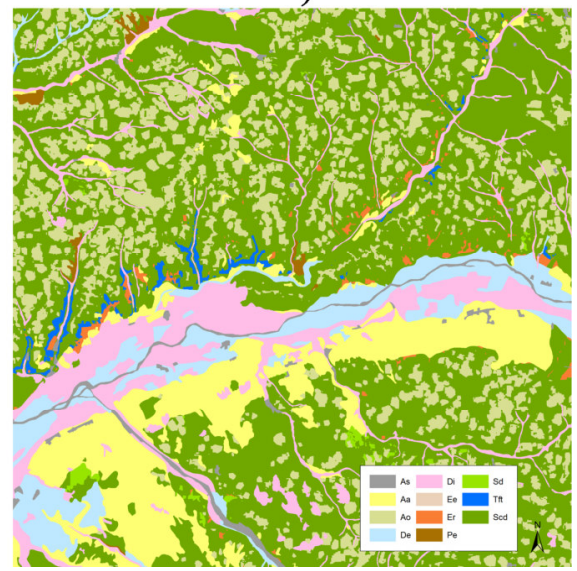

Figura 6 - Ilustração da carta de características diagnóstico de FERREIRA et al. (2001) (a) e das novas caraterísticas diagnóstico (b) (onde, As é área social, Ao alagamento ocasional, De drenagem externa, Di drenagem interna, Peprofundidade expansível, Scd sem características-diagnóstico, Aa armazenamento de água, Ee espessura efectiva, Er perda de solo, Sd acumulação e Tfr toalha freática superficial)

\section{Aderência do modelo}

\section{Dados e análise estatística}

A aderência à realidade, do modelo desenvolvido, foi testada com dados de parcelas permanentes de povoamentos de sobreiro da zona de estudo, pela sua relação com um parâmetro que traduz a qualidade da estação, peso anidro de cortiça por unidade superfície descortiçada, PCM $^{2}$ (MONTERO, 1988). Os dados provêm de 153 parcelas permanentes instaladas pela equipa dos Departamentos de Fitotecnia e Engenharia Rural da Universidade de Évora, com o apoio dos empresários das seguintes explorações: Herdade da Machoqueira do Grou, 66 parcelas, e Herdade de Madeiros, 3 parcelas, ZIF da Calha do Grou, descortiçamento em 1996 a 2001 e 2011, respectivamente; Herdade de Barbas, 3 parcelas, e Herdade do pé d'Erra, 3 parcelas, ZIF da Ribeira da Erra, descortiçamento em 2004 e 2010, respectivamente; Herdade de Vale Mouro, 3 parcelas, ZIF da Ribeira do Divor, descortiçamento em 2006, Herdade do Cascavel, 3 parcelas, ZIF das Ribeiras de Lamarosa e Magos, 
descortiçamento em 2005; Herdade da Torrinha, 3 parcelas, ZIF do Baixo Sorraia, descortiçamento em 2005.

Numa primeira fase às parcelas de inventário, por operações de análise espacial, foram associados os dados de inventário ao nível da árvore, nomeadamente $\mathrm{PCM}^{2}$, e os das novas, características-diagnóstico (Figura 5). A variável $\mathrm{PCM}^{2} \_$médio é o valor ponderado de $\mathrm{PCM}^{2}$ pela área do polígono da parcela de inventário.

A análise estatística inclui a análise de normalidade e a analise de variância. Foi implementada em SPSS (IBM, 2017) para um nível de significância de 0.5 (MARÔCO, 2018). A normalidade avaliada com o teste de Kolmogorov-Smirnov indica que a distribuição a normal dos dados é rejeitada (HOLLANDER e WOLFE, 1973), então a análise de variância a utilizar deve ser não paramétrica, variância das medianas em vez das médias. A análise de variância foi efectuada com o teste de Kruskal-Wallis e ANOVA não paramétrica (IBM, 2017).

\section{Resultados}

O teste de Kruskal-Wallis para $\mathrm{PCM}^{2}$ das diferentes parcelas rejeitou a igualdade das medianas $(\mathrm{p}=0.000)$, indicando que as diferenças são significativas (KRUSKAL e WALLIS, 1952). Para a discriminação das diferenças entre cada par de novas características diagnóstico a ANOVA não paramétrica (Figura 7 e Quadro 4). $\mathrm{PCM}^{2}$ apresenta o valor mais alto para Ao, seguido por ordem decrescente $\mathrm{Sd}, \mathrm{Scd}, \mathrm{Er}, \mathrm{Tft}$. A hipótese da nulidade para cada par de características-diagnóstico é rejeitada, com uma probabilidade 95\%, para todos os pares.

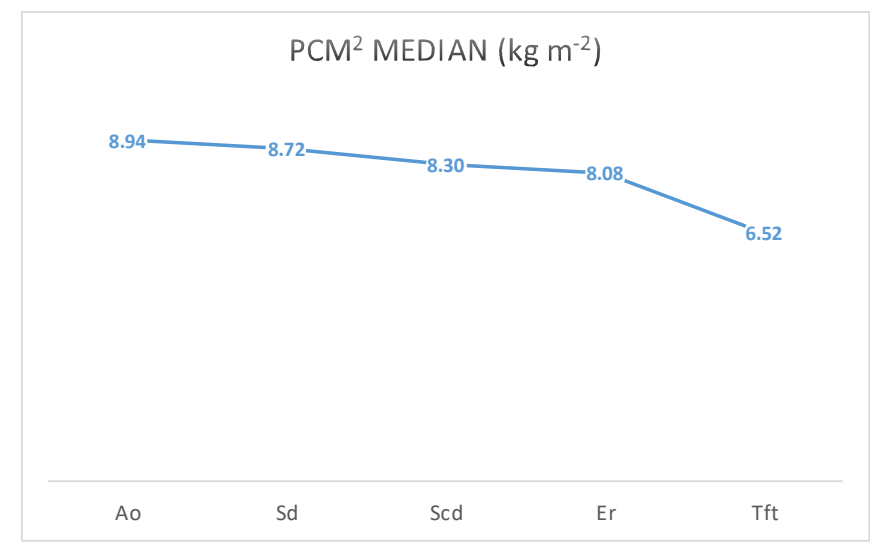

Figura 7 - Características-diagnóstico vs $\mathrm{PCM}^{2}$ 
Quadro 4 - Comparações múltiplas das novas características diagnóstico (Kruskal-Wallis)

\begin{tabular}{|c|c|c|c|c|c|}
\hline $\begin{array}{c}\text { Amostr_1- } \\
\text { Amostra_2 }\end{array}$ & $\begin{array}{c}\text { Teste } \\
\text { Estatístico }\end{array}$ & $\begin{array}{c}\text { Desvio } \\
\text { Padrão } \\
\text { do Erro }\end{array}$ & $\begin{array}{c}\text { Desvio } \\
\text { Padrão } \\
\text { do Teste }\end{array}$ & Significância & $\begin{array}{c}\text { Hipótese da } \\
\text { Nulidade }\end{array}$ \\
\hline Tft-Er & $-29,046.770$ & $1,742.540$ & -16.669 & 0.000 & rejeitada \\
\hline Tft-Scd & $39,032.310$ & $1,730.372$ & 22.557 & 0.000 & rejeitada \\
\hline Tft-Sd & $49,946.176$ & $1,798.595$ & 27.770 & 0.000 & rejeitada \\
\hline Tft-Ao & $58,330.933$ & $2,067.914$ & 28.208 & 0.000 & rejeitada \\
\hline Er-Scd & $9,985.540$ & 300.461 & 33.234 & 0.000 & rejeitada \\
\hline Er-Sd & $29,899.406$ & 564.333 & 37.034 & 0.000 & rejeitada \\
\hline Er-Ao & $29,284.162$ & $1,171.476$ & 24.998 & 0.000 & rejeitada \\
\hline Scd-Sd & $-10,913.866$ & 525.558 & -20.766 & 0.000 & rejeitada \\
\hline Scd-Ao & $-19,298.622$ & $1,153.298$ & -16.733 & 0.000 & rejeitada \\
\hline Sd-Ao & -8384.757 & $1,248.315$ & -6.717 & 0.000 & rejeitada \\
\hline
\end{tabular}

\section{Discussão}

Segundo DINIS (2014) o sobreiro tem um sistema radical dimórfico, um primeiro nível cerca de $0.40 \mathrm{~m}$ de profundidade, composto por raízes finas, com desenvolvimento horizontal e um segundo nível por raiz de desenvolvimento vertical, até cerca de $1.2 \mathrm{~m}$ de profundidade. DAVID et al. (2013) referem que valores de cerca de 1 metro e 4.5 metros respectivamente, podendo o desenvolvimento horizontal exceder a projecção da copa, sendo limitado por mobilizações do solo. DINIS (2014) indica que a ligação deste sistema dimórfico com o freático, é conseguida a partir do primeiro nível por raízes secundárias que se desenvolvem verticalmente, principalmente na periferia da copa (zona de gotejo). O segundo nível vai ligar ao freático mais profundo, podendo existir ligação entre raízes da mesma árvore ou com as de árvores vizinhas (DINIS, 2014).

Foi provado (DAVID et al., 2013; KURZ-BESSON et al., 2006; NADEZHDINA et al., 2008) que o sobreiro movimenta água de zonas mais profundas com maior potencial para as camadas superficiais com menor potencial (redistribuição hidráulica), durante o Verão e o inverso durante o Inverno, quando a relação de potencial de água no solo se inverte, usando o sistema radical como conduta. Este mecanismo complementa a redistribuição da água no solo por ascensão capilar e o armazenamento em profundidade, mantendo em actividade o nível 
superior do sistema e a absorção de nutrientes, que nos solos da área de estudo, estão concentrados no primeiro nível (DAVID et al., 2013; KURZ-BESSON et al., 2006; NADEZHDINA et al., 2008).

O abastecimento em água é a partir do solo do primeiro nível do sistema radical, enquanto o seu potencial for superior ao do freático, caso contrário o abastecimento a partir deste torna-se relevante. As restrições de solo ao desenvolvimento em profundidade da raiz limitam a absorção de água e de nutrientes (DINIS, 2014).

Tendo em conta que o valor de $\mathrm{PCM}^{2}$ é determinado em árvores adultas e considerando em conjunto a informação acima mencionada, os valores de $\mathrm{PCM}^{2}$, decrescem, sempre com diferenças significativas entre medianas (Quadro 4), de Ao, Sd, Scd, Er e Tft, onde se observa o menor valor (Figura 7). A profundidade do solo, Ao, e o fraco escoamento superficial, Sd, com capacidade de armazenamento de água, são factores propiciam um largo volume de solo disponível para o sistema radical, e consequentemente a redistribuição hidráulica, expresso em valores mais elevados de $\mathrm{PCM}^{2}$.

O efeito da erosão na profundidade ao material originário consolidado, $\mathrm{Er}$, a presença de descontinuidade textural, que propiciam a formação de toalhas freáticas temporárias, originando ciclo estacionais de excesso e falta de água, Tft, são factores que limitam o volume de solo disponível e o desenvolvimento do sistema radical e acesso ao freático (Quadro 2), sendo expresso em valores $\mathrm{PCM}^{2}$ mais baixos, sendo, no entanto, o efeito da drenagem interna, Tft, mais limitativo. O volume de solo e capacidade de armazenamento de águas disponíveis na Scd, não potenciados por sedimentação e escoamento superficial, resultam num valor intermédio de $\mathrm{PCM}^{2}$ (Figura 7).

O volume de solo disponível para o desenvolvimento de raízes e a maior disponibilidade água no solo seguido, traduzem-se em valores mais elevados de $\mathrm{PCM}^{2}$, sendo os valores mais baixos os correspondentes redução do volume explorável pelas raízes devido a material originário consolidado e deficiente drenagem interna: Ao - relevo aplanado, perfil profundo, disponibilidade de água; Sd - reduzido escoamento superficial e o material originário não consolidado, propiciam volume de solo disponível; Er - perfil delgado, material originário consolidado e a prevalência da perda de solo sobre a deposição, diminui o volume de solo e água disponíveis; Tft - relevo aplanado, com fraco potencial de escoamento, associado à presença toalhas freáticas temporárias, potencia ciclos excesso e escassez de água no solo; Scd - mantém a produtividade da estação. 


\section{Conclusões}

A equação de formação de solo, (Eq. 1), identifica as variáveis independentes, factores de estado, factores de formação do solo. A metodologia para sua solução, baseiam-se nas localizações de estações onde todos os factores se mantêm contantes menos um, identificando a sua influência na formação da unidade de solo, taxon (DOKUCHAEV, 1899; JENNY, 1941). As cartas interpretativas de uso do solo indicam a adequação da cada unidade do solo, taxon, a dada utilização e identificam características transversais a diferentes taxa, características-diagnóstico, que determinam o seu comportamento e tipo de utilização (FERREIRA et al., 2001). Na zona de estudo, como já foi referido, são constantes todos os factores de estado excepto o factor material originário (p) e relevo (r). O sobreiro ocorre predominantemente nas encostas e vales, rareando nas encostas mais erosionadas e no topo, devido à degradação do solo (GOMES, 1969). Os algoritmos de base topográfica, EHE e DEP, identificando estas posições na paisagem, permitem reclassificar a característica diagnóstico Scd, que representa mais de $76 \%$ da área de estudo em cinco, característicasdiagnóstico. A qualidade da estação, que avalia a potencialidade produtiva do local, solo e clima, é representada para o sobreiro de cortiça amadia pelo $\mathrm{PCM}^{2}$ (MONTERO, 1988). A análise estatística, ANOVA não paramétrica, indica uma boa aderência da metodologia de identificação das características-diagnóstico, nomeadamente dos algoritmos de base topográfica, à qualidade da estação para o sobreiro, avaliada pelo $\mathrm{PCM}^{2}$, nível de significância de $95 \%$, e sua consequente relação com o potencial produtivo do solo.

\section{Referências bibliográficas}

BALL, G.H., HALL, D.J., 1965. Isodata: a method of data analysis ls and pattern classification. Stanford Research Institute, Menlo Park, United States. Office of Naval Research. Information Sciences Branch, 61 pp..

Carta Militar de Portugal, Série M888 - 1/25 000 CIGeo. Centro de Informação Geoespacial do Exército.

CIDFCIBCS, 2015. Plano Intermunicipal de Defesa de Floresta Contra Incêndios, 2011-2015. Comissão Intermunicipal de Defesa da Floresta Contra Incêndios de Benavente, Coruche e Salvaterra de Magos, 112 pp.. 
DAVID, T.S., PINTO, C.A., NADEZHDINA, N., KURZ-BESSON, C., HENRIQUES, M.O., QUILHÓ, T., CERMAK, J., CHAVES, M.M., PEREIRA, J.S., DAVID, J.S., 2013. Root Functioning, Tree Water Use and Hydraulic Redistribution in Quercus suber Trees: A Modeling Approach Based on Root Sap Flow. Forest Ecology and Management 307: 136-146.

DINIS, C.O., 2014. Cork Oak (Quercus suber L.) Root System Structural-Functional 3D Approach. Tese de Doutoramento, Universidade de Évora, 172 pp..

DOKUCHAEV, V.V., 1899. Report to the Transcauca Slan Statistical Committee on Land Evaluation in General and Especially for the Transcaucasia. Horizontal and Vertical Soil Zones, 1899 in Tipogr. kantselyarii Glavnonachal'stvuyushchego gra-zhdanskoi chast'yu na Kavkaze, Tiflis.

ESRI, 1999/2017. ArcGIS desktop: release 10. Environmental Systems Research Institute, Redlands.

HOLLANDER, M., WOLFE, D.A., 1973. Nonparametric statistical methods. John Wiley\& Sons, 819 pp.

FERREIRA, A.G., GONÇALVES, A.C., PINHEIRO, A.C., GOMES, C.P., ILHÉU, M., NEVES, N., RIBEIRO, N.A., SANTOS, P., 2001. Plano Específico de Ordenamento Florestal para o Alentejo. Alfredo Gonçalves Ferreira e Ana Cristina Gonçalves (Eds.). Universidade de Évora, 200 pp.

GOMES, A.M.A., 1969. Fomento da Arborização nos Terrenos Particulares. Fundação Calouste Gulbenkian, 395 pp..

IBM, 2017. IBM SPSS Statistics for Windows, Version 25.0. Armonk, NY: IBM Corp.

JENNY, H., 1941. Factors of Soil Formation a System of Quantitative Pedology. Mac-Graw-Hill, $271 \mathrm{pp}$.

JENNY, H., 1983. The soil resource. Origin and behaviour. Ecological Studies 37. SpringerVerlag, 377 pp..

JENSON, S.K., DOMINGUE, J.O., 1988. Extracting Topographic Structure from Digital Elevation Data for Geographic Information System Analysis. Photogrammetric Engineering and Remote Sensing 54(11): 1593-1600.

KOHNKE, H., BERTRAND, A.R., 1959. Soil conservation. McGraw-Hill book Company, 298 pp..

KURZ-BESSON, C., OTIENO, D., LOBO do VALE, R., SIEGWOL, R., SCHMIDT, M., HERD, A., NOGUEIRA, C., DAVID, T.S., DAVID, J.S., TENHUNEN, J., PEREIRA, J.S., CHAVES, M.M., 2006. Hydraulic Lift in Cork Oak Trees in a Savannah-Type Mediterranean Ecosystem and its Contribution to the Local Water Balance. Plant and Soil 282: 361-378.

KRUSKAL, W.H., WALLIS, W.A., 1952. Use of Ranks in One-Criterion Variance Analysis. Journal of the American Statistical Association 47(260): 583-621.

MERRELL, R., DIAZ, D., 2015. Comparison of Data Mining Methods on Different Applications: Clustering and Classification Methods. Information. Science. Letters 4(2):61-66.

MARÔCO, J., 2018. Análise Estatística com o SPSS Statistics. $7^{a}$ edição. Report Number, 1013 pp.. 
MONTERO, G.G., 1988. Modelos para cuantificar la produccion de corcho enalcornocales (Quercus suberL.) enfuncion de la calidad deestacion y de los tratamientos selvícolas. Dissertacion doctoral, Instituto Nacional de Investigaciones Agrarias, 103 pp..

NADEZHDINA, N., FERREIRA, M.I., SILVA, R., PACHECO, C.A., 2008. Seasonal Variation of Water Uptake of a Quercus suber Tree in Central Portugal. Plant and Soil 305 (1-2): 105-119.

NEVES, N., FERREIRA, A.G., GONÇALVES, A.C., CANCELA d'ÁBREU, A., RAMOS, I.J., FREIRA, M., GUIOMAR, N., 2001. Toolbox EHE - Erosão Hídrica Estrutural. DGOTDU/Universidade de Évora, 19 pp..

ÖZHAN, S., BALCI, A.N., ÖZYUVACI, N., HIZAL, A., GÖKBULAK, F., SERENGIL, Y., 2005. Cover and management factors for the Universal Soil-Loss. Equation for forest ecosystems in the Marmara region, Turkey. Forest Ecology and Management 214: 118-123.

RICHARDSON, B., SKINNER, M.F., WEST, G., 1999. The role of forest productivity in defining the sustainability of plantation forests in New Zealand. Forest Ecology and Management 122: 125-137.

SCHOENHOLTZ, S.H., MIEGROET, H., BURGER, J.A., 2000. A review of chemical and physical properties as indicators of forest soil quality: challenges and opportunities. Forest Ecology and Management 138: 335-356.

SILVA, J.R.M., 1999. Susceptibilidade do solo à erosão hídrica. Tese de Doutoramento, Universidade de Évora, 176 pp..

UNAC, 2013. Guia de Comercialização de Cortiça no Campo. União da Floresta Mediterrânea, 33 pp..

WAHBA, G., 1990. Spline models for Observational data. Society for Industrial and Mathematics, 169 pp..

WISCHMEIER, W.H., SMITH, D.D., 1978. Predicting rainfall erosion losses - a guide to conservation planning. U.S. Department of Agriculture, Agriculture Handbook $\mathrm{n}^{\circ} 537$, 58 pp.. 